\title{
REVIEW
}

\section{Is Plant Sterols a Good Strategy to Lower Cholesterol?}

\author{
Yuwei Chen ${ }^{1}$, Yongbo She ${ }^{2}$, Ramandeep Kaur ${ }^{2}, \mathrm{Na} \mathrm{Guo}^{3}$, Xiaohua Zhang ${ }^{3}$, \\ Ruisan Zhang ${ }^{3}$, and Xingchun Gou ${ }^{3 *}$ \\ ${ }^{1}$ Shaanxi Key Laboratory of Brain Disorders, Department of Public Health, Xi'an Medical University, No.1 Xingwang Road, Xi'an 710021, CHINA \\ ${ }^{2}$ Department of Food and Human Nutritional Sciences, The University of Manitoba, Winnipeg, Manitoba, CANADA \\ ${ }^{3}$ Shaanxi Key Laboratory of Brain Disorders \& Institute of Basic Medical Sciences \& Institute of Basic and Translational Medcine, Xi'an Medical \\ University, No.1 Xingwang Road, Xi'an 710021, CHINA
}

\begin{abstract}
Cardiovascular disease (CVD) has emerged as the leading cause of dealth worldwide today. Lowering circulating total cholesterol (TC) and low density lipoprotein cholesterol (LDL-C) is one of the most effective approaches of CVD prevention. Dietary guidelines and health organizations approved using plant sterols (PS) as the alternative to conventional method in attenuating circulating TC and LDL-C levels and risk of CVD. However, current findings apprear to be controversial on the efficacy of PS. Giving the rise of the field "Nutrigenetics", single nucleotide polymorphisms (SNPs) such as CYP7A1-rs3808607 have been identified that strongly associate with cholesterol metabolism in response to PS intake, towards causing inter-individual variations. This review article aims to discuss the efficacy of dietary PS in managing cholesterol levels based on findings from recent studies. The scope includes reviewing evidence on supporting the efficacy, the metabolic claims, inter-individual variations as well as sitosterolemia associated with PS intake.
\end{abstract}

Key words: plant sterols, cardiovascular disease, nutrigenetics, TC/LDL-C, cholesterol

\section{Introduction}

CVD is recognized as the leading cause of death worldwide, especially in western societies ${ }^{1)}$. In North America, CVD is a leading cause of mortality and morbidity and representing a big economic burden on the direct and indirect cost of the health care system. Recent data published in 2012 showed that approximately 68,342 of all deaths happened per year in Canada due to CVD. Although, the death rate has decreased by $1.9 \%$ from the year 2008 to 2009, still, it is the second major cause of death in Canada ${ }^{2}$. However, it was estimated in 2011 that there are 300 million patients with CVD, and 3 million reported death cases each year in China ${ }^{3)}$. Elevated serum TC, LDL-C levels, low high-density lipoprotein (HDL-C) levels, and high triglycerides (TGs) levels are major lipid biomarkers that contribute to the development of atherosclerosis and therefore, leading to $\mathrm{CVD}^{4)}$.

Health Canada recommended 2 g/day intake of PS which helps to lower the cholesterol levels in human. The reduc- tion in serum lipid profile plays a role in the prevention of $\mathrm{CVD}^{5)}$. PS are used as a non-pharmacological therapy approach for cholesterol lowering by incorporating them into various foods. Intake of PS-enriched foods or supplements is considered a good therapeutic treatment to reduce the risk of $\mathrm{CVD}^{6)}$. It has been shown that intake of PS effectively lowers serum TC and LDL-C concentrations ${ }^{7)}$. Most of the studies have shown that PS may significantly lower serum LDL-C concentration by an average of $8-10 \%$ or 0.31-0.34 mmol/L regardless of gender, age, ethnic background, and body weight ${ }^{8,9)}$. In spite of the clear-cut LDL-C lowering effect of regular PS intake, some health concerns have been raised towards the very high intake of PS-enriched foods. The current review will discuss whether the use of PS is a good strategy to lower cholesterol or not.

\footnotetext{
* Correspondence to: Xingchun Gou, Shaanxi Key Laboratory of Brain Disorders \& Institute of Basic Medical Sciences \& Institute of Basic and Translational Medcine, Xi'an Medical University, No.1 Xingwang Road, Xi'an 710021, CHINA

E-mail: gouxingchun@189.cn

Accepted May 22, 2019 (received for review April 29, 2019)

Journal of Oleo Science ISSN 1345-8957 print / ISSN 1347-3352 online

http://www.jstage.jst.go.jp/browse/jos/ http://mc.manusriptcentral.com/jjocs
} 


\section{Methodology}

Pubmed, Scopus, Research gate and Google Scholar were chosen as serach engines for searching literatures. The terms used for search including PS, plant stanol, phytosterol, phytostanol, sitosterolemia along with terms of serum lipid, TC, LDL-C, HDL-C, TGs. The search preferred to focus primarily on human studies; however, this review chose one animal study as well. The search was limited to peer-reviewed literatures (clinical trials, meta-analysis, review article) published within the last 15 years.

In inclusion criteria, studies used PS as the active treatments were chosen. After reviewing several review papers, meta-analysis, and peer-reviewed articles, some of current clinical trials and review literature were included. The studies were published within last 10 years and focused on using clinical trials with adequate population size and less confounding factors.

\section{Discussion}

\subsection{Chemical composition and dietary sources of PS}

PS and their saturated derivatives plant stanols are collectively called phytosterols. Phytosterols are bioactive components found in plants. These components cannot be synthesized by the human body and therefore, must be obtained from the $\operatorname{diet}^{10)}$. PS are structurally similar to cholesterol, which is found in animal origin; however, they are different from cholesterol in the structure of their side chains. These side chains are differed by having an extra ethyl group in sitosterol and stigmasterol; or a methyl group in campesterol at C-24 of their side chain, whereas stigmasterol is different from sitosterol by having a double bond on the side chain ${ }^{11)}$. Over 250 phytosterols have been identified in free and esterified form and can be divided into sterols and stanols based on their degree of saturation $^{12)}$. Sitosterol, stigmasterol, and campesterol are three most abundant PS found in diet and constitutes 65\%, 30\%, and $3 \%$ of total dietary sterol intakes, respectively ${ }^{13)}$. Si- tosterol is the most abundant PS in diet ${ }^{14)}$. Moreover, 5 $\alpha$-saturated derivatives plant stanols such as cholestanol, campestanol, and sitostanol are major stanol derivatives of $\mathrm{PS}^{11)}$. The natural sources of PS include vegetable oils, whole grains, nuts, fruits, and vegetables. Vegetable oils and their products are the richest natural source of PS, which can be found in free and esterfied forms. However, cereals, vegetables, and fruits are not rich in PS but can also be considered as significant sources of $\mathrm{PS}^{15)}$.

\subsection{Mechanism for cholesterol lowering by detary PS}

The most striking differences between PS and cholesterol in human metabolism including de novo synthesis, intestinal uptake, and biliary secretion ${ }^{11)}$. A primary mechanism of PS is inhibiting the cholesterol absorption in the gastrointestinal tract. In the digestion process, cholesterol gets solubilized by action of bile acids and mixed into the micellar apartment, and absorbed into enterocytes by NiemannPick C1 Like 1 (NPC1L1) ${ }^{17)}$. In the enterocytes, absorbed cholesterol and PS are esterified with acyl-CoA cholesterol acyltransferase-2(ACAT2). The unabsorbed cholesterol excretes out through feces, whereas sterol esters further assimilated with apolipoprotein B and TGs and packed into chylomicrons and then released into the lymph to be moved to the liver ${ }^{18)}$. Unesterified PS and cholesterol pumped back into the intestine by $A B C G 5$ and $A B C G 8$ at the hepatobiliary interface. The absorption efficiency of PS $(<2 \%)$ and plant stanols $(<0.2 \%)$ is 500 fold or 10,000 fold lower as compared to cholesterol (approximately $50 \%)$, and both equally reduce the cholesterol levels ${ }^{11}$. However, O'Neill reported that plant stanols maintained their cholesterol-lowering effects for a long time while the efficacy of sterols decreased after 1 or 2 months ${ }^{19)}$. Overall, PS lower LDL-C levels by reducing the intestinal absorption of cholesterol by $30 \%-50 \%{ }^{16)}$.

\subsection{Current findings on cholesterol lowering by PS}

The dietary intake of naturally occurring PS has a modest effect in lowering serum LDL-C concentrations.

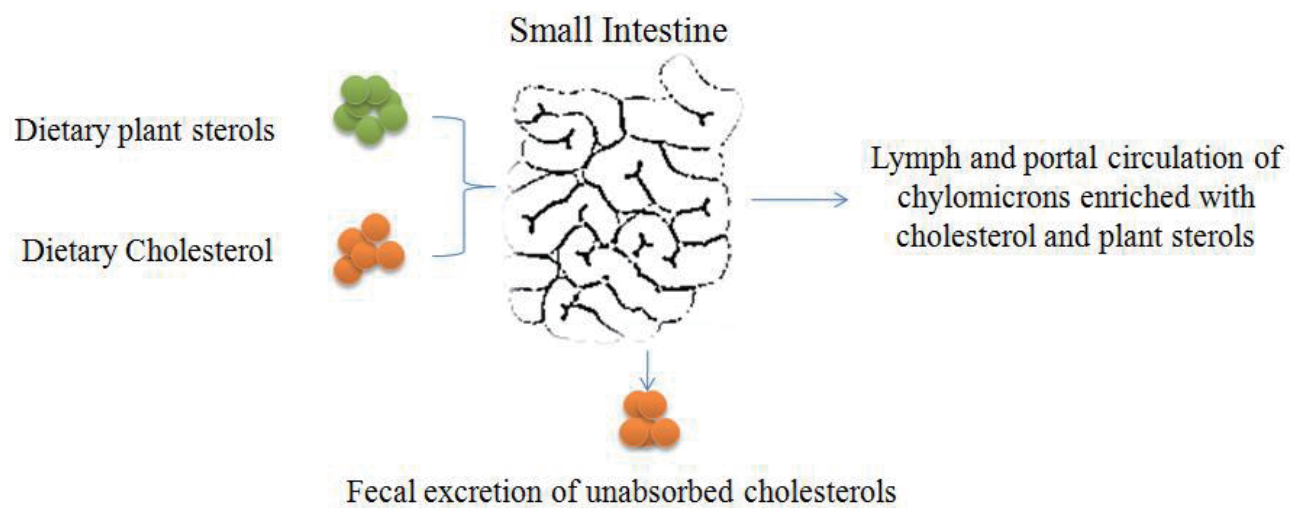

Fig. 1 Proposed mechasims of choletserol lowering by PS. 


\section{Is Plant Sterols a Good Strategy to Lower Cholesterol?}

However, the higher intake of naturally occurring PS (300$400 \mathrm{mg}$ /day) significantly lower serum TC and LDL-C concentrations as compared to lower PS intake which is $<200$ $\mathrm{mg} /$ day $^{20)}$. Thus, after looking at the effects of naturally occurring PS on serum lipid profile, especially LDL-C, PS have been formulated in different foods and food products for many years (since 1995). It has been stated that PS as a part of a heart-healthy diet, play a role as non-pharmacological therapy to lower serum concentrations of TC and LDL-C ${ }^{21)}$. Most of the studies used low fat spread/margarine, yoghurt or small drinks as a medium for esterification of PS into food products ${ }^{9}$. Therefore, many studies have been reported that $2 \mathrm{~g}$ /day PS enriched food and food products or supplements could significantly reduce serum LDL-C concentrations by $8-10 \%$ and have no significant effects on HDL-C. However, there is a dose-dependent relationship between PS and cholesterol-lowering which affects its efficacy. For instance, $0.6 \mathrm{~g} /$ day PS intake lowered serum LDL-C by $6 \%$, whereas $3.3 \mathrm{~g} /$ day intake of PS reduced it by $12 \%{ }^{7)}$. Thus, the effect of cholesterollowering by PS is dose-dependent until it reaches the plateau of $2 \mathrm{~g} /$ day.

The food products enriched with PS usually prescribed to hypercholesterolemic patients. Therefore, many studies used low-fat foods as a medium for the enrichment of PS into them ${ }^{7,22)}$. It has been found that low-fat yogurts significantly lowers serum LDL-C concentrations in moderately hypercholesterolemic patients, compared to low-fat milk ${ }^{23)}$. For instance, the effect of PS was assessed in hypercholesterolemia patients for 30 days. Subjects were served with four different products (papaya fruit bar, soy milk, yogurt, and flavored milk) supplemented with $2 \%$ PS powder with controls which were served with same items without adding PS. All four PS-enriched food products significantly $(p<0.05)$ reduced serum TC and LDL-C concentrations. Interestingly, there was a great reduction in serum lipid concentrations of TC and LDL-C (8.7\% and $12.6 \%)$ after consumption of soy milk compared to other food products and significantly decreased TGs as well. Thus, this study comes up with the conclusion that PS-enriched soy milk is an effective strategy to lower circulating lipid levels and can reduce intestinal cholesterol absorption in hypercholesterolemic individuals ${ }^{23)}$.

According to the National Cholesterol Education Program Adult Treatment Panel(NCEP ATP) III guidelines, less intake of saturated fatty acids and dietary cholesterol, and more consumption of fruits, vegetables, whole grains, and low-fat dairy products, and recommended 2 g/day intake of PS and plant stanols helps in the prevention of $\mathrm{CVD}^{5)}$. Therefore, some studies used low-fat foods to incorporate them with $\mathrm{PS}^{6,24)}$. However, one study found the dose-response relationship of EPA and DHA on several low doses with $2.5 \mathrm{~g}$ /day PS gave a better reduction in TGs and LDL-C concentrations ${ }^{25)}$. Based on that, a recent interven- tion trial demonstrated that consumption of low-fat spread delivering low dose of eicosapentaenoic acid (EPA) + docosahexaenoic acid (DHA) from fish oil significantly lower both TGs (10.6\%) and serum LDL-C (5.2\%) concentrations and thus helps to reduce the risk of $\mathrm{CVD}^{24)}$. However, no significant effect of PS on serum HDL-C was observed. Considering the mechanism behind TGs lowering, PS, EPA, and DHA inhibit hepatic TGs synthesis, decrease VLDL synthesis and secretion, and increase plasma lipoprotein lipase (LPL) activity ${ }^{26)}$. The consumption of PS and $\mathrm{EPA}+\mathrm{DHA}$ also have beneficial effects on lipoprotein metabolism as they significantly reduced non HDL-C concentrations, which could suppress the VLDL-C production. However, some possible factors might affect the efficacy of PS including high saturated spread used for intervention and $\mathrm{DHA}^{27)}$.

\subsection{Challenges regard to PS on cholesterol lowering}

Although it has been suggested a strong efficacy of PS supplementation in lowering circulating TC and LDL-C levels as well as the risk of CVD. However, large variations also exist in current findings and the efficacy of PS has been continually challenged. For instance, a newly published study reported that a daily intake of $3 \mathrm{~g}$ PS esters failed to attenuate neither serum TC nor LCL-C levels in 16 subjects $^{28)}$. Similarly, the randomized crossover trial found that $2 \mathrm{~g}$ /day PS supplementation failed to modulate LDL-C levels in 49 subjects despite a reduction on cholesterol absorption was observed ${ }^{29)}$. However, Alphonse and his colleagues identified huge inter-individual variations of the study subjects in response to PS in a manner that almost half of the subjects responded adversely, whereas other half responded positively ${ }^{29)}$. The inter-individual variation was thought due to poor compliance of participants to dietary treatment or poor quality control of the study; however, individual's endogenous lipid metabolism seems also contributes to this. To support this notion, Rideout et al analyzed results from clinical studies previously published and conducted a follow-up animal study ${ }^{30)}$. They concluded that PS failed to attenuate circulating TC and LDL-C levels in subjects who have high basal de novo cholesterol synthesis, compared to those who have low synthesis rate ${ }^{30)}$. To further delineate the causations and underlying mechanisms, recent research interest has been directed towards studying genes, giving the rise of the field "nutrigenetics".

De Castro-Oros first found that CYP7A1-rs3808607 polymorphisms that strongly associated with cholesterol levels in response to PS treatment ${ }^{31)}$. Soon after this finding, Mackay and his colleagues revealed that CYP7A1rs3808607 G allele carriers are responders to PS, whereas $\mathrm{T} / \mathrm{T}$ homozygous are non-responders ${ }^{32)}$. CYP7A1 encodes the gene for bile acid synthesis from cholesterol pool, whereas $\mathrm{G}$ allele is believed to enhance the gene expres- 
Y. Chen, Y. She, R. Kaur et al.

Table 1 Summary of current findings of PS on circulating lipids profile.

\begin{tabular}{|c|c|c|}
\hline Dose of PS & Action of PS on lipids profile & Reference \\
\hline $0.6 \mathrm{~g} /$ day PS & Lowers LDL-C by $6 \%$ & 7 \\
\hline $3.3 \mathrm{~g} /$ day PS & Lowers LDL-C by $12 \%$ & 7 \\
\hline $\begin{array}{l}\text { 2\% PS powder (Reducol }{ }^{\mathrm{TM}} \text { original Powder) with } \\
\text { papaya fruit bar, soy milk, yogurt and flavored milk }\end{array}$ & $\begin{array}{l}\text { All four products lowers LDL-C by } 12.6 \% \text {, TC by } \\
\qquad 8.7 \%\end{array}$ & 23 \\
\hline 2 g/day PS with DHA and EPA & Lowers LDL-C by $5.2 \%$ and TGs by $10.6 \%$ & 24 \\
\hline $3 \mathrm{~g} /$ day PS ester & No effect on TC and LDL-C & 28 \\
\hline $\begin{array}{c}2 \mathrm{~g} / \text { day PS (55\% } \beta \text {-sitosterol, } 25 \% \text { campesterol, } \\
20 \% \text { stigmasterol })\end{array}$ & No effect on LDL-C & 29 \\
\hline $2 \mathrm{~g} /$ day PS & $\begin{array}{c}\text { Failed to lower TC and LDL-C for high basal de } \\
\text { novo cholesterol synthesis subjects }\end{array}$ & 30 \\
\hline $2 \mathrm{~g} /$ day PS & $\begin{array}{l}\text { Failed to lower LDL-C for CYP7A1-rs3808607 T/T } \\
\text { homozygous }\end{array}$ & 32 \\
\hline
\end{tabular}

sion and increasing bile acid synthesis while increasing cholesterol absorption ${ }^{32)}$. Considering the mechanism of PS in lowering circulating cholesterol levels, enhanced bile acid synthesis and cholesterol absorption by G allele may yield a greater TC and LDL-C reductions. However, to delineate the causation and mechanism clearly, more studies are needed further to validate current finding. In addition to the $C Y 7 A 1$ polymorphism, $A P O E$ polymorphisms that causing different $A P O E$ isoforms ( $\left(22, e 3, e^{4}\right)$; however, was also found to be strongly associated with TC, LDL-C levels in response to $\mathrm{PS}^{32}$. Although a conclusion cannot be made due to limited evidence are available, current findings suggested a strong association between inter-individual variations of TC/LDL-C levels in response to PS and genetics. Therefore, using PS in managing circulating cholesterol levels and lowering risk of CVD may not work for everyone.

On the other hand, sitosterolemia is the rare disease that patients developed abnormally high levels of PS in blood and tissues. The disease is caused by mutation on ATP binding cassette transporter genes, the $A B C G 5$ or $A B C G 8^{333}$. The mutations would result the body failed to pump PS back into intestine and excrete via bile. Thus, dietary PS accumulated in circulating blood and tissues, which would further cause severe consequences. Studies have shown that sitosterolemia strongly associated with premature atherosclerosis, and there are several cases reported in regard to sitosterolemia globally in the past ten years $^{33)}$. The common clinical features of these reported sitosterolemia are including but not limited to elevated levels of circulating PS, skin problems and atherosclerosis that lead to fatal $\mathrm{CVD}^{33}$. The study also reported that sitosterolemia heterozygous who have either $A B C G 5$ or $A B C G 8 \mathrm{mu}-$ tation were found to have higher serum PS levels than control subjects after $2 \mathrm{~g} /$ day PS treatment ${ }^{34)}$. Thus, using PS as the strategy to lower circulating cholesterol levels in the cluster of subjects appears to be impossible. Instead, these patients were instructed to take alternatives such as ezetimibe and cholestyramine to lower circulating PS as well as cholesterol levels ${ }^{33)}$.

In addition to the inter-individual variations in response to PS and sitosterolemia as previously mentioned. Study also reported that oxidized PS in human body are also associated with the development of atherosclerosis ${ }^{35)}$. Besides, a recent animal study reported that PS significantly increased body weight and interfered gut microbiome in mice ${ }^{36}$. The study suggested that the novel functional food, PS may alter host microbiome and contribute to weight gain while attenuating cholesterol levels ${ }^{36)}$. The exact mechanisms and causations regard to body weight gain and gut microbiota interference by consuming dietary PS are still not fully understood and well established. However, these findings further suggested the challenges associate with dietary consumption of PS.

\section{Summary and Conclusions}

Although findings suggest that daily intake of $2 \mathrm{~g}$ PS is capable to attenuate TC and LDL-C levels toward lowering the risk of CVD. The health claims have also been approved by several organizations such as Health Canada, Food and Drug Agency of American and European Food Safety Authority. However, current findings are controversial. For instance, genetic-wise inter-individual variations suggest that some subjects are non-responders to PS, whereas the LDL-C and TC can even be increased by consuming $2 \mathrm{~g} /$ day PS. In addition, sitosterolemia, circulating oxidized PS and weight gain associated with PS consumption are also a concern. Thus, using PS to lower cholesterol levels may not the best strategy in some subjects. Patients who are hypo-responders or sitosterolemic in response to PS ought to seek alternatives to manage cholesterol levels and risk 
of CVD.

\section{Acknowledgements}

This work was supported by the Project of Shannxi Key Laboratory of Brain Disorders (No.18NBZD05), the Young Scholars Research Funding of Xi'an Medical University (No.2018QN04), the National Natural Science Foundation of China (No.81810188).

\section{Conflicts of Interest}

All authours declare have no conflict of interest.

\section{References}

1) Rader, D.J.; Daugherty, A. Translating molecular discoveries into new therapies for atherosclerosis. $\mathrm{Na}$ ture 451, 904 (2008).

2) Mortality, summary list of causes.[Internet]. Statistics Canada. https://www150.statcan.gc.ca/n1/en/ catalogue/84F0209X2009000. Accessed 25 July 2012.

3) Li, H.; Ge, J. Cardiovascular diseases in China: Current status and future perspectives. IJC Heart \& Vasculature 6, 25-31 (2015).

4) Libby, P. Inflammation in atherosclerosis. Arterioscler. Thromb. Vasc. Biol. 32, 2045-2051 (2012).

5) Expert Panel on Detection, Evaluation, and Treatment of High Blood Cholesterol in Adults. Executive summary of the third report of the National Cholesterol Education Program, NCEP(Adult Treatment Panel III). JAMA 285, 2486-2497 (2001).

6) Trautwein, E.A.; Koppenol, W.P.; Arienne, D.J.; Harry, H.; Vermeer, M. A.; Manny, N. Plant sterols lower LDLcholesterol and triglycerides in dyslipidemic individuals with or at risk of developing type 2 diabetes; a randomized, double-blind, placebo-controlled study. Nutr. Diabetes 8, 30 (2018).

7) Ras, R.T.; Geleijnse, J.M.; Trautwein, E.A. LDL-cholesterol-lowering effect of plant sterols and stanols across different dose ranges: a meta-analysis of randomised controlled studies. Br. J. Nutr. 112, 214-219(2014).

8) Demonty, I.; Ras, R.T.; Van, d.K.H.C.M.; Duchateau, G.S.M.J.E.; Meijer, L.; Zock, P.L. Continuous dose-response relationship of the LDL-cholesterol-lowering effect of phytosterol intake. J. Nutr. 139, 271-284 (2008).

9) Gylling, H.; Plat J.; Turley S.; Henry N. Plant sterols and plant stanols in the management of dyslipidaemia and prevention of cardiovascular disease. Atherosclerosis 232, 346-360 (2014).
10) Racette, S.B.1.; Lin, X.; Ma, L.; Ostlund, R.E.Jr. Natural dietary phytosterols. J. AOAC Int. 98, 679-684 (2015).

11) Helena, G,; Piia, S. Phytosterols, phytostanols, and lipoprotein metabolism. Nutrients 7, 7965-7977 (2015).

12) Moreau, R.A.; Whitaker, B.D.; Hicks, K.B. Phytosterols, phytostanols, and their conjugates in foods: structural diversity, quantitative analysis, and health-promoting uses. Prog. Lipid Res. 41, 457-500 (2002).

13) Gupta, A.K.; Savopoulos, C.G.; Ahuja, J; Hatzitolios, A.I. Role of phytosterols in lipid-lowering: current perspectives. QJM 104, 301-308(2011).

14) Escolà-Gil, J.C.; Quesada, H,; Julve, J.; Martín-Campos, J.M.; Cedó, L.; Blanco-Vaca, F. Sitosterolemia: diagnosis, investigation, and management. Curr. Atheroscler. Rep. 16, 424-570 (2014).

15) Ogbe, R.J.; Ochalefu, D.O.; Mafulul, S.G. A review on dietary phytosterols: Their occurrence, metabolism and health benefits. Asian J. Plant Sci. Res. 5, 10-21 (2015).

16) Abumweis, S.S.; Marinangeli, C.P.F.; Frohlich, J.; Jones, P.J.H. Implementing phytosterols into medical practice as a cholesterol-lowering strategy: overview of efficacy, effectiveness, and safety. Can. J. Cardiol. 30, 1225-1232 (2014).

17) Davis, H.R.; Zhu, L. J.; Hoos, L.M.; Tetzloff, G.; Maguire, M.; Liu, J. Niemann-pick c1 like 1 (npc1l1) is the intestinal phytosterol and cholesterol transporter and a key modulator of whole-body cholesterol homeostasis. J. Biol. Chem. 279, 33586-33592(2004).

18) Gylling, H.K.; Hallikainen, M.; Vidgren, H.; Agren, J.; Miettinen, T.A. Ester percentages of plant sterols and cholesterol in chylomicrons and vldl of humans with low and high sterol absorption. Atherosclerosis 187, 150-152 (2006).

19) O’Neill, F.H.; Brynes, A.; Mandeno, R.; Rendell, N.; Taylor, G.; Seed, M. Comparison of the effects of dietary plant sterol and stanol esters on lipid metabolism. Nutr. Metab. Cardiovasc. Dis. 14, 133-142 (2004).

20) Klingberg, S.1.; Ellegård, L.; Johansson, I.; Hallmans, G.; Weinehall, L.; Andersson, H.; Winkvist, A. Inverse relation between dietary intake of naturally occurring plant sterols and serum cholesterol in northern sweden. Am. J. Clin. Nutr. 87, 993-1001 (2008).

21) Simonen, P.; Sittiwet, C.; Nissinen, M.J.; Gylling, H. Phytosterol consumption and coronary artery disease. in Handbook of nutrition in heart health. Wageningen Academic Publishers. pp. 1553-1561 (2017).

22) Ras, R.T.; Hiemstra, H.; Lin, Y.; Vermeer, M.A.; Duchateau, G.S.M.J.E.; Trautwein, E. A. Consumption of plant sterol-enriched foods and effects on plasma plant sterol concentrations - a meta-analysis of randomized controlled studies. Atherosclerosis 230, 336- 
346 (2013).

23) Penchalaraju, M.; Kuna, A.; Supraja, P.S.T.; Ramprasath, V.; Jones, P.J.H. Cholesterol-lowering efficacy of plant sterol-enriched flavored milk, yogurt, fruit bar, and soya milk in mild hypercholesterolemic Indian subjects. Clin. J. Nutr. Diet. 1, 1-6(2018).

24) Blom, W.A.M.; Koppenol, W.P.; Hiemstra, H.; Stojakovic, T.; Scharnagl, H.; Trautwein, E.A. A low-fat spread with added plant sterols and fish omega-3 fatty acids lowers serum triglyceride and LDL-cholesterol concentrations in individuals with modest hypercholesterolaemia and hypertriglyceridaemia. Eur. J. Nutr. 1-10 (2018).

25）Ras, R.T.; Demonty, I.; Zebregs, Y.E.M.P.;Quadt, J.F.A.; Olsson, J.; Trautwein, E.A. Low doses of eicosapentaenoic acid and docosahexaenoic acid from fish oil dosedependently decrease serum triglyceride concentrations in the presence of plant sterols in hypercholesterolemic men and women. J. Nutr. 144, 1564-1570(2014).

26) Oscarsson, J.; Hurt-Camejo, E. Omega-3 fatty acids eicosapentaenoic acid and docosahexaenoic acid and their mechanisms of action on apolipoprotein b-containing lipoproteins in humans: a review. Lipids Health Dis. 16, 149 (2017).

27) Wei, M.Y.; Jacobson, T.A. Effects of eicosapentaenoic acid versus docosahexaenoic acid on serum lipids: a systematic review and meta-analysis. Curr. Atheroscler. Rep. 13, 474-483 (2011).

28) Weingärtner, O.; Bogeski, I.; Kummerow, C.; Schirmer, S.H.; Vanmierlo, T.; Wagenpfeil, G.; Hoth, M.; Böhm, M,; Lütjohann, D.; Laufs U. Plant sterol ester diet supplementation increases serum plant sterols and markers of cholesterol synthesis, but has no effect on total cholesterol levels. J. Steroid Biochem. Mol. Biol. 169, 219-225 (2016).

29) Alphonse, P.A.S.; Ramprasath, V.; Jones, P.J.H. Effect of dietary cholesterol and plant sterol consumption on plasma lipid responsiveness and cholesterol trafficking in healthy individuals. Br. J. Nutr. 117, 56-66 (2017).

30) Rideout, T.C.; Harding, S.V.; Mackay, D.; Abumweis, S.S.; Jones, P.J. High basal fractional cholesterol synthesis is associated with nonresponse of plasma LDL cholesterol to plant sterol therapy. Am. J. Clin. Nutr. 92, 41-46 (2010).

31) De Castro-Orós, I,; Pampín, S.; Cofán, M,; Mozas, P.; Pintó, X.; Salas-Salvadó, J. Rodríguez-Rey, J.C.; Ros, E,; Civeira, F.; Pocoví, M. Promoter variant-204A>C of the cholesterol $7 \alpha$-hydroxylase gene: Association with response to plant sterols in humans and increased transcriptional activity in transfected HepG2 cells. Clin. Nutr. 30, 239-246 (2011).

32) Mackay, D.S.; Eck, P.K.; Gebauer, S.K.; Baer, D.J.; Jones, P.J. Cyp7a1-rs3808607 and apoe isoform associate with LDL cholesterol lowering after plant sterol consumption in a randomized clinical trial. Am. J. Clin. Nutr. 102, 951-957 (2015).

33) Myrie, S.B.; Othman, R.A.; Ajagbe, B.O. Plant sterols, stanols, and sitosterolemia. J. AOAC Int. 98, 716-723 (2015).

34) Kratz, M.; Kannenberg, F.; Gramenz, E.; Berning, B.; Trautwein, E.; Assmann, G.; Rust, S. Similar serum plant sterol responses of human subjects heterozygous for a mutation causing sitosterolemia and controls to diets enriched in plant sterols or stanols. Eur. J. Clin. Nutr. 61, 896-905 (2007).

35) Köhler, J.; Teupser, D.; Elsässer, A.; Weingärtner, O. Plant sterol enriched functional food and atherosclerosis. Br. J. Pharmacol. 174, 1281-1289 (2017).

36) Ryan, P.M.; London, L.E.; Bjorndahl, T.C.; Mandal, R. Murphy, K; Fitzgerald, G.F.; Shanahan, F.; Ross, R.; Wishart. D.S. Caplice, N.M.; Stanton, C. Microbiome and metabolome modifying effects of several cardiovascular disease interventions in apo-E - / - mice. $M i-$ crobiome 5, 30 (2017). 